\title{
An extremely luminous X-ray outburst at the birth of a supernova
}

A. M. Soderberg ${ }^{1,2}$, E. Berger ${ }^{1,2}$, K. L. Page ${ }^{3}$, P. Schady $^{4}$, J. Parrent ${ }^{5}$,

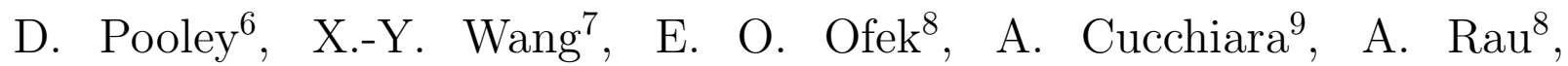
E. $\operatorname{Waxman}^{10}$, J. D. Simon ${ }^{8}$, D. C.-J. Bock ${ }^{11}$, P. A. Milne ${ }^{12}$, M. J. Page ${ }^{4}$, J. C.Barentine ${ }^{13}$, S. D. Barthelmy ${ }^{14}$, A. P. Beardmore ${ }^{3}$, M. F. Bietenholz ${ }^{15,16}$, P. Brown ${ }^{9}$, A. Burrows ${ }^{1}$, D. N. Burrows ${ }^{9}$, G. Byrngelson ${ }^{17}$, S. B. Cenko ${ }^{18}$, P. Chandra ${ }^{19}$ J. R. Cummings ${ }^{20}$, D. B. Fox ${ }^{9}$, A. Gal-Yam ${ }^{10}$, N. Gehrels ${ }^{20}$, S. Immler ${ }^{20}$, M. Kasliwal ${ }^{8}$, A. K. H. Kong ${ }^{21}$, H. A. Krimm ${ }^{20,22}$, S. R. Kulkarni ${ }^{8}$ T. J. Maccarone ${ }^{23}$, P. Mészáros ${ }^{9}$, E. $\mathrm{Nakar}^{24}$, P. T. O'Brien ${ }^{3}$, R. A. Overzier ${ }^{25}$, M. de Pasquale ${ }^{4}$, J. Racusin ${ }^{9}$, N. Rea ${ }^{23}$, and D. G. York ${ }^{26}$

${ }^{1}$ Department of Astrophysical Sciences, Princeton University, Ivy Lane, Princeton, NJ 08544, USA

${ }^{2}$ Carnegie Observatories, 813 Santa Barbara St., Pasadena, CA 91101, USA

${ }^{3}$ Department of Physics and Astronomy, University of Leicester, Leicester LE1 7RH, UK

${ }^{4}$ Mullard Space Sci. Lab., Univ. College London, Holmbury St. Mary, Dorking, Surrey RH5 6NT, UK

${ }^{5}$ Physics and Astronomy Department Dartmouth College, Hanover, NH 03755, USA

${ }^{6}$ Astronomy Department, University of Wisconsin, 475 North Charter Street, Madison, WI 53706, USA

7 Department of Astronomy, Nanjing University, Nanjing 210093, China

8 Department of Astronomy, 105-24, California Institute of Technology, Pasadena, CA 91125, USA

${ }^{9}$ Dept. of Astronomy and Astrophysics, Pennsylvania State University, University Park, PA 16802, USA

${ }^{10}$ Faculty of Physics, Weizmann Institute of Science, Rehovot 76100, Israel

${ }^{11}$ Radio Astronomy Laboratory, University of California, Berkeley, CA 94720, USA

12 Steward Observatory, University of Arizona, 933 North Cherry Avenue, Tucson, AZ 85721, USA

${ }^{13}$ Department of Astronomy, University of Texas at Austin, Austin, TX 78712, USA

${ }^{14}$ NASA Goddard Space Flight Center, Greenbelt, MD 20771, USA

${ }^{15}$ Department of Physics and Astronomy, York University, Toronto, ON M3J 1P3, Canada

${ }^{16}$ Hartebeestehoek Radio Observatory, PO Box 443, Krugersdorp, 1740, South Africa

17 Dept. of Physics and Astronomy, Clemson University, Clemson, South Carolina 29634, USA

18 Space Radiation Laboratory 220-47, California Institute of Technology, Pasadena, CA 91125, USA

19 Department of Astronomy, University of Virginia, P.O. Box 400325, Charlottesville, VA 22904, USA

${ }^{20}$ CRESST and NASA Goddard Space Flight Center, Greenbelt, MD 20771, USA

${ }^{21}$ Institute of Astronomy and Dept. of Physics, National Tsing Hua University, Hsinchu, Taiwan

${ }^{22}$ Universities Space Research Association, 10211 Wincopin Circle, \# 500, Columbia, MD 21044, USA

${ }^{23}$ School of Physics and Astronomy, University of Southampton, Southampton SO17 1BJ, UK

24 Theoretical Astrophysics 130-33, California Institute of Technology, Pasadena, CA 91125, USA

${ }^{25}$ Max-Planck-Institut fur Astrophysik, D-85748 Garching, DE

${ }^{26}$ Dept. of Astronomy and Astrophysics, Univ. of Chicago, 5640 S. Ellis Avenue, Chicago, IL 60637, USA 
Massive stars end their short lives in spectacular explosions, supernovae, that synthesize new elements and drive galaxy evolution. Throughout history supernovae were discovered chiefly through their delayed optical light, preventing observations in the first moments (hours to days) following the explosion. As a result, the progenitors of some supernovae and the events leading up to their violent demise remain intensely debated. Here we report the serendipitous discovery of a supernova at the time of explosion, marked by an extremely luminous X-ray outburst. We attribute the outburst to the break-out of the supernova shock-wave from the progenitor, and show that the inferred rate of such events agrees with that of all core-collapse supernovae. We forecast that future wide-field X-ray surveys will catch hundreds of supernovae each year in the act of explosion, and thereby enable crucial neutrino and gravitational wave detections that may ultimately unravel the explosion mechanism.

Stars more massive than about eight times the mass of the Sun meet their death in cataclysmic explosions termed supernovae (SNe). These explosions give birth to the most extreme compact objects - neutron stars and black holes - and enrich their environments with heavy elements. It is generally accepted that SNe are triggered when the stellar core runs out of fuel for nuclear burning and thus collapses under its own gravity (see Ref. 1 and references therein). As the collapsing core rebounds, it generates a shock-wave that propagates through and explodes the star.

The resulting explosion ejects several solar masses of stellar material with a mean velocity ${ }^{2}$ of about $10^{4} \mathrm{~km} \mathrm{~s}^{-1}$, or a kinetic energy of about $10^{51} \mathrm{erg}$. Less than a solar mass of ${ }^{56} \mathrm{Ni}$ is synthesized in the explosion, but its subsequent radioactive decay powers ${ }^{1}$ the luminous optical light observed to peak 1 to 3 weeks after the explosion. It is through this delayed signature that SNe have been discovered both historically and in modern searches.

While the general picture of core-collapse has been recognized for many years, the details of the explosion remain unclear and most SN simulations fail to produce an explosion. The gaps in our understanding are due to the absence of detailed observations in the first days after the explosion, and the related difficulty in detecting the weak neutrino ${ }^{3}$ and gravitational wave signatures of the explosion. These signals offer a direct view of the explosion mechanism but require the discovery of SNe at the time of explosion.

In this paper we describe our serendipitous discovery of an extremely luminous Xray outburst that marks the birth of a SN of Type Ibc. Prompt bursts of X-ray and/or ultraviolet (UV) emission have been theorized ${ }^{4,5}$ to accompany the break-out of the SN 
shock-wave through the stellar surface, but their short durations (just seconds to hours) and the lack of sensitive wide-field X-ray and UV searches have prevented their discovery until now.

Our detection enables an unprecedented early and detailed view of the $\mathrm{SN}$, allowing us to infer ${ }^{6}$ the radius of the progenitor star, its mass loss in the final hours prior to the explosion, and the speed of the shock as it explodes the star. Drawing on optical, UV, radio, and X-ray observations we show that the progenitor was compact $\left(R_{*} \approx 10^{11} \mathrm{~cm}\right)$ and stripped of its outer Hydrogen envelope by a strong and steady stellar wind. These properties are consistent ${ }^{7}$ with those of Wolf-Rayet (WR) stars, the favored ${ }^{8}$ progenitors of Type Ibc SNe.

Wolf-Rayet stars are also argued ${ }^{9}$ to give rise to gamma-ray bursts (GRBs), a related but rare class of explosions characterized by highly-collimated relativistic jets. Our observations, however, indicate an ordinary spherical and non-relativistic explosion and we firmly rule out a GRB connection.

Most important, the inferred rate of X-ray outbursts indicates that all core-collapse SNe produce detectable shock break-out emission. Thus, future wide-field X-ray surveys will uncover hundreds of SNe each year at the time of explosion, providing the long-awaited temporal and positional triggers for neutrino and gravitational wave searches.

\section{Discovery of the X-ray Outburst}

On 2008 Jan 9 at 13:32:49 UT, we serendipitously discovered an extremely bright Xray transient during a scheduled Swift X-ray Telescope (XRT) observation of the galaxy NGC $2770(d=27 \mathrm{Mpc})$. Previous XRT observations of the field just two days earlier revealed no pre-existing source at this location. The transient, hereafter designated as X-ray outburst (XRO) 080109, lasted about $400 \mathrm{~s}$, and was coincident with one of the galaxy's spiral arms (Figure 1). From observations described below we determine that XRO 080109 is indeed located in NGC 2770, and we thus adopt this association hereafter.

The temporal evolution is characterized by a fast rise and exponential decay, often observed for a variety of X-ray flare phenomena (Figure 1). We determine the onset of the X-ray emission to be $9_{-8}^{+20} \mathrm{~s}$ before the beginning of the observation, implying an outburst start time of $t_{0} \approx$ Jan $9.564 \mathrm{UT}$. The X-ray spectrum is best fit by a power law $\left[N(E) \propto E^{-\Gamma}\right]$ with a photon index of $\Gamma=2.3 \pm 0.3$, and a hydrogen column density of $N_{H}=6.9_{-1.5}^{+1.8} \times 10^{21} \mathrm{~cm}^{-2}$, in excess of the absorption within the Milky Way (see Suppl. Info.). The inferred unabsorbed peak flux is $F_{X, p} \approx 6.9 \times 10^{-10} \mathrm{erg} \mathrm{cm}^{-2} \mathrm{~s}^{-1}$ $(0.3-10 \mathrm{keV})$. We also measure significant spectral softening during the outburst.

The XRO was in the field of view of the Swift Burst Alert Telescope (BAT; $15-150 \mathrm{keV}$ ) 
$4 \quad$ Soderberg et al.

beginning 30 min before and continuing throughout the outburst but no $\gamma$-ray counterpart was detected. Thus, the outburst was not a GRB (see also Suppl. Info.). Integrating over the duration of the outburst, we place a limit on the gamma-ray fluence of $f_{\gamma} \lesssim 8 \times 10^{-8}$ erg $\mathrm{cm}^{-2}(3 \sigma)$, a factor of three times higher than an extrapolation of the X-ray spectrum to the BAT energy band.

The total energy of the outburst is thus $E_{X} \approx 2 \times 10^{46} \mathrm{erg}$, at least three orders of magnitude lower ${ }^{10}$ than GRBs. The peak luminosity is $L_{X, p} \approx 6.1 \times 10^{43} \mathrm{erg} \mathrm{s}^{-1}$, several orders of magnitude larger than the Eddington luminosity (the maximum luminosity for a spherically-accreting source) of a solar mass object, outbursts from Ultra-luminous X-ray sources and Type I X-ray bursts. In summary, the properties of XRO 080109 are distinct from those of all known X-ray transients.

\section{The Birth of a Supernova}

Simultaneous observations of the field with the co-aligned Ultraviolet/Optical Telescope (UVOT) on-board Swift showed no evidence for a contemporaneous counterpart. However, UVOT observations just $1.4 \mathrm{hr}$ after the outburst revealed ${ }^{11}$ a brightening UV/optical counterpart. Subsequent ground-based optical observations also uncovered $^{11-13}$ a coincident source.

We promptly obtained optical spectroscopy of the counterpart with the Gemini North 8-m telescope beginning $1.74 \mathrm{~d}$ after the outburst (Figure 2). The spectrum is characterized by a smooth continuum with narrow absorption lines of Na I $\lambda \lambda 5890,5896$ at the redshift of NGC 2770. More importantly, we note broad absorption features near 5200 and $5700 \AA$ and a drop-off beyond $7000 \AA$, strongly suggestive of a young SN. Subsequent observations confirmed these spectral characteristics, ${ }^{11,14}$ and the transient was classified ${ }^{11,15}$ as Type Ibc SN 2008D based on the lack of hydrogen and weak silicon features.

Thanks to the prompt X-ray discovery, the temporal coverage of our optical spectra exceeds those of most SNe, rivaling even the best-studied GRB-associated SNe (GRB-SNe), and SN 1987A (Figure 2). We see a clear evolution from a mostly featureless continuum to broad absorption lines, and finally to strong absorption features with moderate widths. Moreover, our spectra reveal the emergence of strong He I features within a few days of the outburst (see also Ref. 16). Thus, SN 2008D is a He-rich SN Ibc, unlike ${ }^{17}$ GRB-SNe. Observations at high spectral resolution further reveal significant host galaxy extinction, with $A_{V} \approx 1.2-2.5 \mathrm{mag}$ (see Suppl. Info.).

The well-sampled UV/optical light curves in ten broadband filters $(2000-10,000 \AA)$ exhibit two distinct components (Figure 3). First, a UV-dominated component that peaks about a day after the X-ray outburst, and which is similar to very early observations ${ }^{18}$ of 
GRB-SN 2006aj. The second component is significantly redder and peaks on a timescale of about 20 days, consistent with observations of all SNe Ibc. Accounting for an extinction of $A_{V}=1.9 \mathrm{mag}$ (Figure 3), the absolute peak brightness of the second component is $M_{V} \approx-16.7 \mathrm{mag}$, at the low end of the distribution ${ }^{19}$ for SNe Ibc and GRB-SNe.

\section{A Shock Break-out Origin}

Since some SNe Ibc harbor GRB jets, we investigate the possibility that the XRO is produced by a relativistic outflow. In this scenario, the X-ray flux and simultaneous upper limits in the $\mathrm{UV} /$ optical require the outflow to be ultra-relativistic with a bulk Lorentz factor, $\gamma \approx 90$, but its radius to be only $R \approx 10^{10} \mathrm{~cm}$; here $\gamma \equiv\left(1-\beta^{2}\right)^{-1 / 2}$ and $\beta \equiv v / c$, where $v$ is the outflow velocity and $c$ is the speed of light. However, given the observed duration of the outburst, we expect ${ }^{20} R \approx 4 \gamma^{2} c t \approx 10^{17} \mathrm{~cm}$, indicating that the relativistic outflow scenario is not self-consistent (see Suppl. Info. for details).

We are left with a trans- or non-relativistic origin for the outburst and consider SN shock break-out as a natural scenario. The break-out is defined by the transition from a radiation-mediated to a collisional (or collisionless ${ }^{21}$ ) shock as the optical depth of the outflow decreases to unity. Such a transition has long been predicted ${ }^{4,5}$ to produce strong, thermal UV/X-ray emission at the time of explosion. A non-thermal component at higher energies may be produced ${ }^{22}$ by multiple scatterings of the photons between the ejecta and dense circumstellar medium (bulk Comptonization).

We attribute the observed non-thermal outburst to Comptonized emission from shock break-out indicating that the associated thermal component must lie below the XRT low energy cutoff, $\sim 0.1 \mathrm{keV}$. With the reasonable assumption that the energy in the thermal $\left(E_{\mathrm{th}}\right)$ and Comptonized components is comparable, we constrain ${ }^{6}$ the radius at which shock break-out occurs to $R_{s b o} \gtrsim 7 \times 10^{11}(T / 0.1 \mathrm{keV})^{-4 / 7}\left(E_{X} / 2 \times 10^{46} \mathrm{erg}\right)^{3 / 7} \mathrm{~cm}$. This is consistent with a simple estimate derived from the rise time of the outburst, $R_{s b o}=c \delta t \sim$ $10^{12} \mathrm{~cm}$, and larger than the typical radii of WR stars, ${ }^{23} R_{*} \sim 10^{11}$. We therefore attribute the delayed shock break-out to the presence of a dense stellar wind, similar ${ }^{6,18}$ to the case of GRB-SN 2006aj.

The shock velocity at break-out is ${ }^{6}(\gamma \beta) \lesssim 1.1$ and the outflow is thus trans-relativistic as expected $^{24}$ for a compact progenitor. Using these constraints, the inferred optical depth of the ejecta to thermal X-rays is $\tau_{\mathrm{ej}} \approx 1.5\left(E_{X} / 2 \times 10^{46} \mathrm{erg}\right)\left(R_{s b o} / 7 \times 10^{11} \mathrm{~cm}\right)^{-2}(\gamma-1)^{-1} \approx$ 3, and Comptonization is thus efficient, confirming our model. Equally important, as the ejecta expand outward the optical depth of the stellar wind decreases and the spectrum of the Comptonized emission is expected ${ }^{22}$ to soften, in agreement with the observed trend.

The shock break-out emission traces the wind mass loss rate of the progenitor, $\dot{M}$, in the 
Soderberg et al.

final hours leading up to the explosion. The inferred density indicates $\dot{M} \approx 4 \pi v_{w} R_{s b o} / \kappa \approx$ $10^{-5} \mathrm{M}_{\odot} \mathrm{yr}^{-1}$; here $\kappa \approx 0.4 \mathrm{~cm}^{2} \mathrm{~g}^{-1}$ is the Thomson opacity for an ionized hydrogen wind and $v_{w} \approx 10^{3} \mathrm{~cm} \mathrm{~s}^{-1}$ is the typical ${ }^{7}$ wind velocity for WR stars. The mass loss rate is consistent ${ }^{7}$ with the average values inferred for Galactic WR stars, and along with the inferred compact stellar radius and the lack of hydrogen features, leads us to conclude that the progenitor was a WR star.

\section{Two UV/optical Emission Components}

The early UV/optical emission ( $t \lesssim 3$ day) appears to be a distinct component based on its different temporal behavior and bluer colors (Figure 3 ). We attribute this early emission to cooling of the outer stellar envelope following the passage of the shock through the star and its subsequent break-out (marked by the X-ray outburst). The expected blackbody radiation is characterized ${ }^{6}$ by the photospheric radius and temperature, which evolve as $R_{\mathrm{ph}} \propto t^{0.8}$ and $T_{\mathrm{ph}} \propto t^{-0.5}$, and depend on the total ejecta kinetic energy $\left(E_{K}\right)$ and mass $\left(M_{\mathrm{ej}}\right)$, and on the stellar radius prior to the explosion $\left(R_{*}\right)$.

The model light curves provide a good fit to the early UV/optical data (Figure 3). The implied stellar radius is $R_{*} \approx 7 \times 10^{10} \mathrm{~cm}$, consistent with that expected ${ }^{23}$ for a Wolf-Rayet progenitor. Moreover, this value is smaller than the shock break-out radius, confirming our earlier inference that the break-out occurs in the extended stellar wind.

The ratio of $E_{K}$ and $M_{\mathrm{ej}}$ also determines the shape of the main SN light curve (e.g., Ref. 25), and the mass of ${ }^{56} \mathrm{Ni}$ synthesized in the explosion $\left(M_{\mathrm{Ni}}\right)$ determines ${ }^{26}$ its peak optical luminosity. To break the degeneracy between $E_{K}$ and $M_{\mathrm{ej}}$, we measure the photospheric velocity from the optical spectra at maximum light, $v_{\mathrm{ph}}=0.3\left(E_{K} / M_{\mathrm{ej}}\right)^{1 / 2} \approx$ $11,500 \mathrm{~km} \mathrm{~s}^{-1}$, comparable to that of ordinary SNe Ibc, but somewhat slower ${ }^{17}$ than GRB-SNe (Figure 2 and Suppl. Info). We find that both light curve components are selfconsistently fit with $E_{K} \approx(2-4) \times 10^{51} \mathrm{erg}, M_{\mathrm{ej}} \approx 3-5 \mathrm{M}_{\odot}$, and $M_{\mathrm{Ni}} \approx 0.05-0.1 \mathrm{M}_{\odot}$ (Figure 3).

\section{Long-lived X-ray and Radio Emission}

While UV/optical observations probe the bulk material, radio and X-ray emission trace fast ejecta. Our Swift follow-up observations of the XRO revealed fainter X-ray emission several hours after the explosion with $L_{X} \approx 2 \times 10^{40} \mathrm{erg} \mathrm{s}^{-1}(t \approx 0.2 \mathrm{~d})$. This emission exceeds the extrapolation of the outburst by many orders of magnitude, indicating that it is powered by a different mechanism. Using a high angular resolution observation from the Chandra X-ray Observatory (CXO) on Jan 19.86 UT we detect the SN with a luminosity, $L_{X}=(1.0 \pm 0.3) \times 10^{39} \mathrm{erg} \mathrm{s}^{-1}(0.3-10 \mathrm{keV})$, and further resolve three nearby sources contained within the 18 arcsec resolution element of XRT. Correcting all XRT observations 
for these sources, we find that the long-lived X-ray emission decays steadily as $F_{X} \propto t^{-0.7}$ (Suppl. Info.).

Using the Very Large Array (VLA) on Jan 12.54 UT, we further discovered a new radio source at the position of the SN that was not present on Jan 7 UT. Follow-up observations were obtained at multiple frequencies between 1.4 and $95 \mathrm{GHz}$ using the VLA, the Combined Array for Research in Millimeter-wave Astronomy (CARMA) and the Very Long Baseline Array (VLBA).

The broadband radio emission on Jan 14 reveals a spectral peak, $\nu_{p} \approx 43 \mathrm{GHz}$, with a flux density, $F_{\nu, p} \approx 4 \mathrm{mJy}$, and a low frequency spectrum, $F_{\nu} \propto \nu^{2.5}$. Subsequent observations show that $\nu_{p}$ cascades to lower frequencies, similar to the evolution observed in other SNe Ibc (e.g., Ref. 27). The passage of $\nu_{p}$ through each frequency produces a light curve peak. We measure $F_{\nu} \propto t^{1.4}$ and $F_{\nu} \propto t^{-1.2}$ for the light curve rise and decline, respectively (Figure 4).

We highlight that our X-ray and radio observations of SN 2008D are the earliest ever obtained for a normal SN Ibc. At $t \approx 10$ days, the X-ray and peak radio luminosities are several orders of magnitude lower ${ }^{28,29}$ than those of GRB afterglows but comparable ${ }^{30,31}$ to those of normal SNe Ibc.

\section{The Properties of the Fast Ejecta}

Radio synchrotron emission is produced ${ }^{32}$ by relativistic electrons accelerated in the SN shock as they gyrate in the amplified magnetic field. Self-absorption suppresses the spectrum below the peak to $F_{\nu} \propto \nu^{2.5}$, in excellent agreement with our observations. In this context, we infer 33,34 the radius of the fast ejecta, using the measured $\nu_{p}$ and $L_{\nu, p}$, to be $R \approx 3 \times 10^{15} \mathrm{~cm}$ at $t \approx 5 \mathrm{~d}$. The implied mean velocity is $\beta \approx 0.25$, clearly ruling out relativistic ejecta.

With this conclusion there are two possibilities for the ejecta dynamics. First, the SN may be in free expansion, $R \propto t$, consistent with observations of SNe Ibc (e.g., Ref. 27). Alternatively, the ejecta may have been relativistic at early time and then rapidly decelerated, leading to $R \propto t^{2 / 3}$. In the latter scenario the dynamics are governed ${ }^{35}$ by the Sedov-Taylor solution. As discussed in the Suppl. Info., the temporal evolution of the radio light curves is clearly inconsistent with the Sedov-Taylor model, ruling out even early relativistic expansion.

Thus, the radio emission is produced by freely expanding ejecta, indicative of the broad velocity structure expected ${ }^{24}$ for ordinary core-collapse SNe. The standard formulation ${ }^{27}$ provides an excellent fit to the data (Figure 4) and indicates that the energy coupled to fast material is $E_{K, R} \approx 10^{48} \mathrm{erg}$, just $0.1 \%$ of the total kinetic energy. Moreover, the 
Soderberg et al.

inferred density profile is $\rho(r) \propto r^{-2}$, as expected for a steady stellar wind. The inferred mass loss rate, $\dot{M} \approx 7 \times 10^{-6} \mathrm{M}_{\odot} \mathrm{yr}^{-1}$, is in agreement with our shock break-out value, indicating stable mass loss in the final $\sim 3 \mathrm{yr}$ to $\sim 3 \mathrm{hr}$ of the progenitor's life.

The radio-emitting electrons also account for the late X-ray emission through their inverse Compton upscattering of the SN optical photons (with a luminosity, $L_{\mathrm{opt}}$ ). The expected $^{6}$ X-ray luminosity is $L_{I C} \approx 3 \times 10^{39}\left(E_{K, R} / 10^{48} \mathrm{erg}\right)\left(L_{\mathrm{opt}} / 10^{42} \mathrm{erg} \mathrm{s}^{-1}\right)(t / 1 \mathrm{~d})^{-2 / 3}$ erg $\mathrm{s}^{-1}$, in excellent agreement with the XRT and CXO observations. We note that the synchrotron contribution in the X-ray band is lower by at least two orders of magnitude.

Finally, we note that neither the late X-ray emission nor the radio emission show evidence for a rising component that could be attributed ${ }^{36}$ to an off-axis GRB jet spreading into our line of sight. This conclusion is also supported by the unresolved size of the radio SN from VLBA observations at $t \approx 1$ month, $R \lesssim 2.4 \times 10^{17} \mathrm{~cm}(3 \sigma)$, which constrains the outflow velocity to be $\gamma \beta \lesssim 3$.

\section{The Rate of XROs}

To estimate the rate of XROs we find that the on-sky effective monitoring time of the XRT from the launch of Swift through Jan 2008, including only those exposures longer than $300 \mathrm{~s}$, is about two years. Along with the XRT field of view (24 arcmin on a side), the number density of $L_{*}$ galaxies ( $\phi \approx 0.05 L_{*} \mathrm{Mpc}^{-3}$ ), and the detectability limit of XRT for events like XRO $080109(d \lesssim 200 \mathrm{Mpc})$, we infer an XRO rate of $\gtrsim 10^{-3} L_{*}^{-1} \mathrm{yr}^{-1}(95 \%$ confidence level); here $L_{*}$ is the characteristic luminosity of galaxies. ${ }^{37}$ This rate is at least an order of magnitude larger than for GRBs. ${ }^{38,39}$ On the other hand, with a core-collapse SN rate ${ }^{40}$ of $10^{-2} \mathrm{~L}_{*} \mathrm{yr}^{-1}$, the probability of detecting at least one XRO if all such SNe produce an outburst is about $50 \%$.

We find a similar agreement with the SN rate using the sensitivity of the BAT. The estimated ${ }^{39}$ peak photon flux of the outburst is $0.03 \mathrm{~cm}^{-2} \mathrm{~s}^{-1}(1-1000 \mathrm{keV})$, which for a $10^{2} \mathrm{~s}$ image trigger ${ }^{41}$ is detectable to about $20 \mathrm{Mpc}$. The BAT on-sky monitoring time of 3 years and the 2 ster field of view thus yield an upper limit on the XRO rate of $\lesssim 10^{5}$ $\mathrm{Gpc}^{-3} \mathrm{yr}^{-1}$, consistent with the core-collapse SN rate ${ }^{42}$ of $6 \times 10^{4} \mathrm{Gpc}^{-3} \mathrm{yr}^{-1}$.

Finally, we note that NGC 2770 hosted an unusually high rate of three SNe Ibc in the past 10 years. However, the galaxy has a typical luminosity $\left(0.3 L_{*}\right)$ and a total star formation rate of only $0.5-1 \mathrm{M}_{\odot} \mathrm{yr}^{-1}$ (see Suppl. Info.), two orders of magnitude lower than the extreme starburst galaxy Arp 220, which has ${ }^{43}$ a SN rate of $4 \pm 2 \mathrm{yr}^{-1}$. The elevated SN rate in NGC 2770, with a chance probability of $\sim 10^{-4}$, may simply be a statistical fluctuation given the sample of $\sim 4 \times 10^{3}$ known SN host galaxies. Alternatively, 
it may point to a recent episode of elevated star formation activity, perhaps triggered by interaction with the companion galaxy NGC $2770 \mathrm{~B}$ at a separation ${ }^{44}$ of only $22 \mathrm{kpc}$.

\section{Implications for Supernova Progenitors}

Our observations probe the explosion ejecta over a wide range in velocity, $\sim 10,000-$ $210,000 \mathrm{~km} \mathrm{~s}^{-1}$. Taken together, the material giving rise to the X-ray outburst, the radio emission, and the optical light traces an ejecta profile of $E_{K} \propto(\gamma \beta)^{-4}$ up to transrelativistic velocities. This profile is in good agreement with theoretical expectations ${ }^{24}$ for a standard hydrodynamic spherical explosion of a compact star, but much steeper ${ }^{39}$ than for relativistic GRB-SNe.

On the other hand, we note the similarity between the shock break-out properties of the He-rich SN 2008D and the He-poor GRB-SN 2006aj, both suggestive of a dense stellar wind around a compact Wolf-Rayet progenitor. In the context of SNe Ibc and GRB progenitors, this provides evidence for continuity (and likely a single progenitor system) between He-rich and He-poor explosions, perhaps including GRBs.

Looking forward, our inference that every core-collapse $\mathrm{SN}$ is marked by an XRO places the discovery and study of SNe on the cusp of a paradigm shift. An all-sky X-ray satellite with a sensitivity similar to that of the Swift/XRT will detect and localize several hundred core-collapse SNe per year, even if they are obscured by dust, at the time of explosion. As we have shown here, this will enable a clear mapping between the properties of the progenitors and those of the SNe. Most important, however, X-ray outbursts will provide an unprecedented positional and temporal trigger for neutrino and gravitational wave detectors (such as IceCube and Advanced LIGO), which may ultimately hold the key to unraveling the mystery of the SN explosion mechanism, and perhaps the identity of the compact remnants.

Received 5 May 2008; Accepted draft.

1. Woosley, S. E. \& Weaver, T. A. The physics of supernova explosions. Ann. Rev. Astr. Ap. 24, 205-253 (1986).

2. Filippenko, A. V. Optical Spectra of Supernovae. Ann. Rev. Astr. Ap. 35, 309-355 (1997).

3. Arnett, W. D., Bahcall, J. N., Kirshner, R. P. \& Woosley, S. E. Supernova 1987A. Ann. Rev. Astr. Ap. 27, 629-700 (1989).

4. Colgate, S. A. Early Gamma Rays from Supernovae. Astrophys. J. 187, 333-336 (1974).

5. Klein, R. I. \& Chevalier, R. A. X-ray bursts from Type II supernovae. Astrophys. J. 223, L109-L112 (1978). 
10 Soderberg et al.

6. Waxman, E., Mészáros, P. \& Campana, S. GRB 060218: A Relativistic Supernova Shock Breakout. Astrophys. J. 667, 351-357 (2007).

7. Cappa, C., Goss, W. M. \& van der Hucht, K. A. A Very Large Array 3.6 Centimeter Continuum Survey of Galactic Wolf-Rayet Stars. Astrophys. J. 127, 2885-2897 (2004)

8. Woosley, S. E., Hegar, A. \& Weaver, T. A. The evolution and explosion of massive stars Astrophys. J. 74, 1015-1071 (2002).

9. MacFadyen, A. I., Woosley, S. E. \& Hegar, A. Supernovae, Jets, and Collapsars. Astrophys. J. 550, 410-425 (2001).

10. Soderberg, A. M., Kulkarni, S. R., Berger, E., Fox, D. W., Sako, M. et al. The sub-energetic $\gamma$-ray burst GRB 031203 as a cosmic analogue to the nearby GRB 980425. Nature 430, 648-650 (2004).

11. Page, K. L., Immler, S., Beardmore, A. P., Burrows, D. N., Gehrels, N. et al. Observations of an X-ray transient in NGC 2770. GCNR 110, 1 (2008).

12. Deng, J. \& Zhu, Y. Bright X-ray transient (a XRF? ) in NGC 2770 - a SN optical counterpart? GRB Coordinates Network 7160, 1 (2008).

13. Valenti, S., Turatto, M., Navasardyan, H., Benetti, S. \& Cappellaro, E. Early OT detection of XRF in NGC 2770 in asiago frames. GRB Coordinates Network 7163, 1 (2008).

14. Malesani, D., Hjorth, J., Jakobsson, P., Vreeswijk, P. M., Thoene, C. C. et al. Transient in NGC 2770: spectroscopic evidence for a SN. GRB Coordinates Network 7169, 1 (2008).

15. Li, W. \& Filippenko, A. V. Supernova 2008D in NGC 2770. Central Bureau Electronic Telegrams 1202, 1 (2008).

16. Modjaz, M., Chornock, R., Foley, R. J., Filippenko, A. V., Li, W. et al. Supernova 2008D in NGC 2770. Central Bureau Electronic Telegrams 1222, 1 (2008).

17. Pian, E., Mazzali, P. A., Masetti, N., Ferrero, P., Klose, S. et al. An optical supernova associated with the X-ray flash XRF 060218. Nature 442, 1011-1013 (2006).

18. Campana, S., Mangano, V., Blustin, A. J., Brown, P., Burrows, D. N. et al. The association of GRB 060218 with a supernova and the evolution of the shock wave. Nature 442, 1008-1010 (2006).

19. Soderberg, A. M., Kulkarni, S. R., Price, P. A., Fox, D. B., Berger, E. et al. An HST Study of the Supernovae Accompanying GRB 040924 and GRB 041006. Astrophys. J. 636, 391-399 (2006). 
20. Sari, R., Piran, T. \& Narayan, R. Spectra and Light Curves of Gamma-Ray Burst Afterglows. Astrophys. J. 497, 17-20 (1998).

21. Waxman, E. \& Loeb, A. TeV Neutrinos and GeV Photons from Shock Breakout in Supernovae. Phys. Rev. Lett. 87, 071101 (2001).

22. Wang, X.-Y., Li, Z., Waxman, E. \& Mészáros, P. Nonthermal Gamma-Ray/X-Ray Flashes from Shock Breakout in Gamma-Ray Burst-Associated Supernovae. Astrophys. J. 664, 10261032 (2007).

23. Moffat, A. F. J., Drissen, L. \& Robert, C. in IAU Colloq. 113: Physics of Luminous Blue Variables (eds Davidson, K., Moffat, A. F. J. \& Lamers, H. J. G. L. M.) 229-237 (1989).

24. Matzner, C. D. \& McKee, C. F. The Expulsion of Stellar Envelopes in Core-Collapse Supernovae. Astrophys. J. 510, 379-403 (1999).

25. Valenti, S., Benetti, S., Cappellaro, E., Patat, F., Mazzali, P. et al. The broad-lined Type Ic supernova 2003jd. Mon. Not. R. astr. Soc. 383, 1485-1500 (2008).

26. Arnett, W. D. Type I supernovae. I - Analytic solutions for the early part of the light curve. Astrophys. J. 253, 785-797 (1982).

27. Soderberg, A. M., Kulkarni, S. R., Berger, E., Chevalier, R. A., Frail, D. A. et al. The Radio and X-Ray-Luminous Type Ibc Supernova 2003L. Astrophys. J. 621, 908-920 (2005).

28. Berger, E., Kulkarni, S. R. \& Frail, D. A. A Standard Kinetic Energy Reservoir in GammaRay Burst Afterglows. Astrophys. J. 590, 379-385 (2003).

29. Frail, D. A., Kulkarni, S. R., Berger, E. \& Wieringa, M. H. A Complete Catalog of Radio Afterglows: The First Five Years Astronom. J. 125, 2299-2306 (2003).

30. Berger, E., Kulkarni, S. R., Frail, D. A. \& Soderberg, A. M. A Radio Survey of Type Ib and Ic Supernovae: Searching for Engine-driven Supernovae. Astrophys. J. 599, 408-418 (2003).

31. Kouveliotou, C., Woosley, S. E., Patel, S. K., Levan, A., Blandford, R. et al. Chandra Observations of the X-Ray Environs of SN 1998bw/GRB 980425. Astrophys. J. 608, 872$882(2004)$.

32. Chevalier, R. A. Self-similar solutions for the interaction of stellar ejecta with an external medium. Astrophys. J. 258, 790-797 (1982).

33. Readhead, A. C. S. Equipartition brightness temperature and the inverse Compton catastrophe. Astrophys. J. 426, 51-59 (1994).

34. Kulkarni, S. R., Frail, D. A., Wieringa, M. H., Ekers, R. D., Sadler, E. M. et al. Radio emission from the unusual supernova 1998bw and its association with the $\gamma$-ray burst of 25 April 1998 Nature 395, 663-669 (1998). 
12 Soderberg et al.

35. Waxman, E. The Nature of GRB 980425 and the Search for Off-Axis Gamma-Ray Burst Signatures in Nearby Type Ib/c Supernova Emission. Astrophys. J. 602, 886-891 (2004).

36. Soderberg, A. M., Nakar, E., Berger, E. \& Kulkarni, S. R. Late-Time Radio Observations of 68 Type Ibc Supernovae: Strong Constraints on Off-Axis Gamma-Ray Bursts. Astrophys. J. 638, 930-937 (2006).

37. Blanton, M. R., Hogg, D. W., Bahcall, N. A., Brinkmann, J., Britton, M. et al. The Galaxy Luminosity Function and Luminosity Density at Redshift $\mathrm{z}=0.1$. Astrophys. J. 592, 819-838 (2003).

38. Schmidt, M. Luminosity Function of Gamma-Ray Bursts Derived without Benefit of Redshifts. Astrophys. J. 552, 36-41 (2001).

39. Soderberg, A. M., Kulkarni, S. R., Nakar, E., Berger, E., Cameron, P. B. et al. Relativistic ejecta from X-ray flash XRF 060218 and the rate of cosmic explosions. Nature 442, 1014-1017 (2006).

40. Cappellaro, E., Evans, R. \& Turatto, M. A new determination of supernova rates and a comparison with indicators for galactic star formation. 351, 459-466 (1999).

41. Band, D. L. Postlaunch Analysis of Swift's Gamma-Ray Burst Detection Sensitivity. Astrophys. J. 644, 378-384 (2006).

42. Dahlen, T., Strolger, L.-G., Riess, A. G., Mobasher, B., Chary, R.-R. et al. High-Redshift Supernova Rates. Astrophys. J. 613, 189-199 (2004).

43. Lonsdale, C. J., Diamond, P. J., Thrall, H., Smith, H. E. \& Lonsdale, C. J. VLBI Images of 49 Radio Supernovae in Arp 220. Astrophys. J. 647, 185-193 (2006).

44. Fynbo, J. P. U., Malesani, D., Augusteijn, T. \& Niemi, S.-M. NGC 2770B has the same redshift as NGC 2770. GRB Coordinates Network 7186, 1 (2008).

Correspondence should be addressed to A. M. Soderberg (e-mail: alicia@astro.princeton.edu).

\section{Acknowledgements}

Based in part on observations obtained at the Gemini Observatory through the Director's Discretionary Time. Gemini is operated by the Association of Universities for Research in Astronomy, Inc., under a cooperative agreement with the NSF on behalf of the Gemini partnership: the National Science Foundation (United States), the Science and Technology Facilities Council (United Kingdom), the National Research Council (Canada), CONICYT 
(Chile), the Australian Research Council (Australia), CNPq (Brazil) and SECYT (Argentina). The VLA is operated by the National Radio Astronomy Observatory, a facility of the National Science Foundation operated under cooperative agreement by Associated Universities, Inc. Some of the data presented herein were obtained at the W. M. Keck Observatory, which is operated as a scientific partnership among the California Institute of Technology, the University of California and the National Aeronautics and Space Administration. The Observatory was made possible by the generous financial support of the W. M. Keck Foundation. AMS acknowledges support by NASA through a Hubble Fellowship grant. 
14 Soderberg et al.

Figure 1: Discovery image and X-ray light curve of XRO 080109/SN 2008D Panel a: X-ray (left) and UV (right) images of the field obtained on 2008 Jan 7 UT during Swift observations of the Type Ibc SN 2007uy. No source is detected at the position of SN 2008D to a limit of $\lesssim 10^{-3}$ counts $\mathrm{s}^{-1}$ in the X-rays and $U \gtrsim 20.3$ mag. Panel $b$ : Repeated UV and X-ray observations of the field from Jan 9 UT during which we serendipitously discovered XRO 080109 and its UV counterpart. The position of XRO 080109 is $\alpha=09^{\mathrm{h}} 09^{\mathrm{m}} 30.70^{\mathrm{s}}$ and $\delta=33^{\circ} 08^{\prime} 19.1^{\prime \prime}$ (J2000) ( \pm 3.5 arcsec), about $9 \mathrm{kpc}$ from the center of NGC 2770. Panel $c$ : X-ray light curve of XRO 080109 in the $0.3-10 \mathrm{keV}$ band. The data were accumulated in the photon counting mode and were processed using version 2.8 of the Swift software package, including the most recent calibration and exposure maps. The high count rate resulted in photon pile-up, which we correct for by fitting a King function profile to the point spread function (PSF) to determine the radial point at which the measured PSF deviates from the model. The counts were extracted using an annular aperture that excluded the affected 4 pixel core of the PSF, and the count rate was corrected according to the model. Using a fast rise, exponential decay model (red curve) we determine the properties of the outburst, in particular its onset time, $t_{0}$, which corresponds to the explosion time of SN2008D. The best-fit parameters are a peak time of $63 \pm 7 \mathrm{~s}$ after the beginning of the observation, an e-folding time of $129 \pm 6 \mathrm{~s}$, and peak count rate of $6.2 \pm 0.4$ counts s ${ }^{-1}$ (90\% confidence level using Cash statistics). The best-fit value of $t_{0}$ is Jan 9 13:32:40 UT (i.e., 9 s before the start of the observation) with a $90 \%$ uncertainty range of 13:32:20 to $13: 32: 48 \mathrm{UT}$.

Figure 2: Optical spectra of XRO 080109/SN 2008D. The observations were performed using the following facilities: The Gemini Multi-Object Spectrograph (GMOS) on the Gemini-North $8 \mathrm{~m}$ telescope (black); the Dual Imaging Spectrograph (DIS) on the Apache Point $3.5 \mathrm{~m}$ telescope (blue); the Double Spectrograph (DBSP) on the Palomar Hale 200-inch telescope (green); and the Low Resolution Spectrograph (LRS) on the Hobby-Eberly $9.2 \mathrm{~m}$ telescope (magenta). The details of the observational setup and the exposure times are provided in the Suppl. Info. The data were reduced using the gemini package within the Image Reduction and Analysis Facility (IRAF) software for the GMOS data. All other observations were reduced using standard packages in IRAF. The SN spectra were extracted from the two-dimensional data using a nearby background region to reduce the contamination from host galaxy emission. Absolute flux calibration was achieved using observations of the standard stars Feige 34 and G191B2B. The spectra are plotted logarithmically in flux units and shifted for clarity. The bottom panel includes a 
model fit to the Jan 25 spectrum using the spectral fitting code SYNOW. We identify several strong features attributed to He I, O I, and Fe II indicating a Type Ibc classification. In addition, we find an absorption feature at $6200 \AA$ that can be identified as Si II or high velocity H I (see Suppl. Info. for details).

Figure 3: Optical and UV light curves of XRO 080109/SN 2008D. Data are from SwiftUVOT (circles), Palomar 60-inch telescope (P60; squares), Gemini/GMOS (diamonds), and the SLOTIS telescope (triangles). Tables summarizing the observations and data analysis are available in the Suppl. Info. The data have not been corrected for host galaxy extinction and have been offset (as labeled) for clarity. We fit the data prior to 3 days with a cooling envelope blackbody emission model ${ }^{6}$ (dashed lines) that accounts for host extinction $\left(A_{V}\right)$. We find a reasonable fit to the data with $R_{*} \approx 10^{11} \mathrm{~cm}$, $E_{K} \approx 2 \times 10^{51} \mathrm{erg}, M_{\mathrm{ej}} \approx 5 \mathrm{M}_{\odot}$ and $A_{V} \approx 1.9 \mathrm{mag}$, consistent with the constraints from the high-resolution optical spectrum. The radius and temperature of the photosphere at $1 \mathrm{~d}$ are $R_{\mathrm{ph}} \approx 3 \times 10^{14} \mathrm{~cm}$ and $T_{\mathrm{ph}} \approx 10^{4} \mathrm{~K}$, respectively. The bottom panel shows the absolute bolometric magnitude light curve (corrected for host extinction), The dashed lines are the same cooling envelope model described above, while the dotted lines are models of supernova emission powered by radioactive decay. The solid lines are combined models taking into account the decay of ${ }^{56} \mathrm{Ni}$ (thin line) and ${ }^{56} \mathrm{Ni}+{ }^{56} \mathrm{Co}$ (thick line). The SN models provide an independent measure of $E_{K}$ and $M_{\mathrm{ej}}$, as well as $M_{\mathrm{Ni}}$ (see Suppl. Info. for a detailed discussion of the models). We find values that are consistent to within $30 \%$ with those inferred from the cooling envelope model.

Figure 4: Radio light curves, spectra, and image of XRO 080109/SN 2008D The radio data from 1.4 to $95 \mathrm{GHz}$ were obtained with the VLA, CARMA, and the VLBA (circles are detections and inverted triangles represent $3 \sigma$ upper limits). The flux measurements and a descripton of the data analysis are provided in the Supp. Info. Panel a: Radio light-curves with a model of synchrotron self-absorbed emission arising ${ }^{27,}$ ? from shocked material surrounding the freely-expanding SN. We adopt a shock compression factor of $\eta=4$ for the post-shock material and assume that the electrons and magnetic fields each contribute $10 \%$ to the total post-shock energy density. The best fit model (solid lines) implies the following physical parameters and temporal evolution: $R \approx 3 \times 10^{15}(t / 5 \mathrm{~d})^{0.9}$ $\mathrm{cm}, E_{K, R} \approx 10^{48}(t / 5 \mathrm{~d})^{0.8} \mathrm{erg}$, and $B \approx 2.4(t / 5 \mathrm{~d})^{-1}$, where $B$ is the magnetic field strength. The implied density profile is $\rho(r) \propto r^{-2}$, as expected for the wind from a massive star. Panel b: Broadband radio spectra. The spectral peak of the radio synchrotron emission cascades to lower frequencies over the course of our follow-up observations with $\nu_{p} \propto t^{-1}$. 
The low frequency turn-over is consistent with expectations for synchrotron self-absorption (grey lines). Panel c: Radio image from a VLBA observation on Feb 8 UT. We place an upper limit on the angular size of the ejecta of 1.2 mas $(3 \sigma)$, corresponding to a physical radius of $\lesssim 2.4 \times 10^{17} \mathrm{~cm}$. This limit is factor of 16 times larger, and therefore consistent with the radius derived from the radio SN model. However, it places a limit of $(\gamma \beta) \lesssim 3$ on the expansion velocity.

Figure 5: Volumetric rate of X-ray outbursts similar to XRO 080109. We use all XRT observations longer than $300 \mathrm{~s}$ along with the field of view (24 arcmin on a side), the number density of $L_{*}$ galaxies $\left(\phi \approx 0.05 L_{*} \mathrm{Mpc}^{-3}\right)$, and the detectability limit of XRT for events like XRO $080109(d \lesssim 200 \mathrm{Mpc})$. The curves indicate the rate $\left(L_{*}^{-1} \mathrm{Mpc}^{-3}\right.$ $\mathrm{yr}^{-1}$ ) inferred from one detection in a total of about 2 years of effective on-sky XRT observations as a function of the distance to which XROs can be detected. Also shown are the rates ${ }^{40}$ of core-collapse SNe (solid horizontal line) and SNe Ibc (dashed horizontal line) as determined from optical SN searches. The rate of events like XRO 080109 is consistent with the core-collapse rate at the $50 \%$ probability level. 

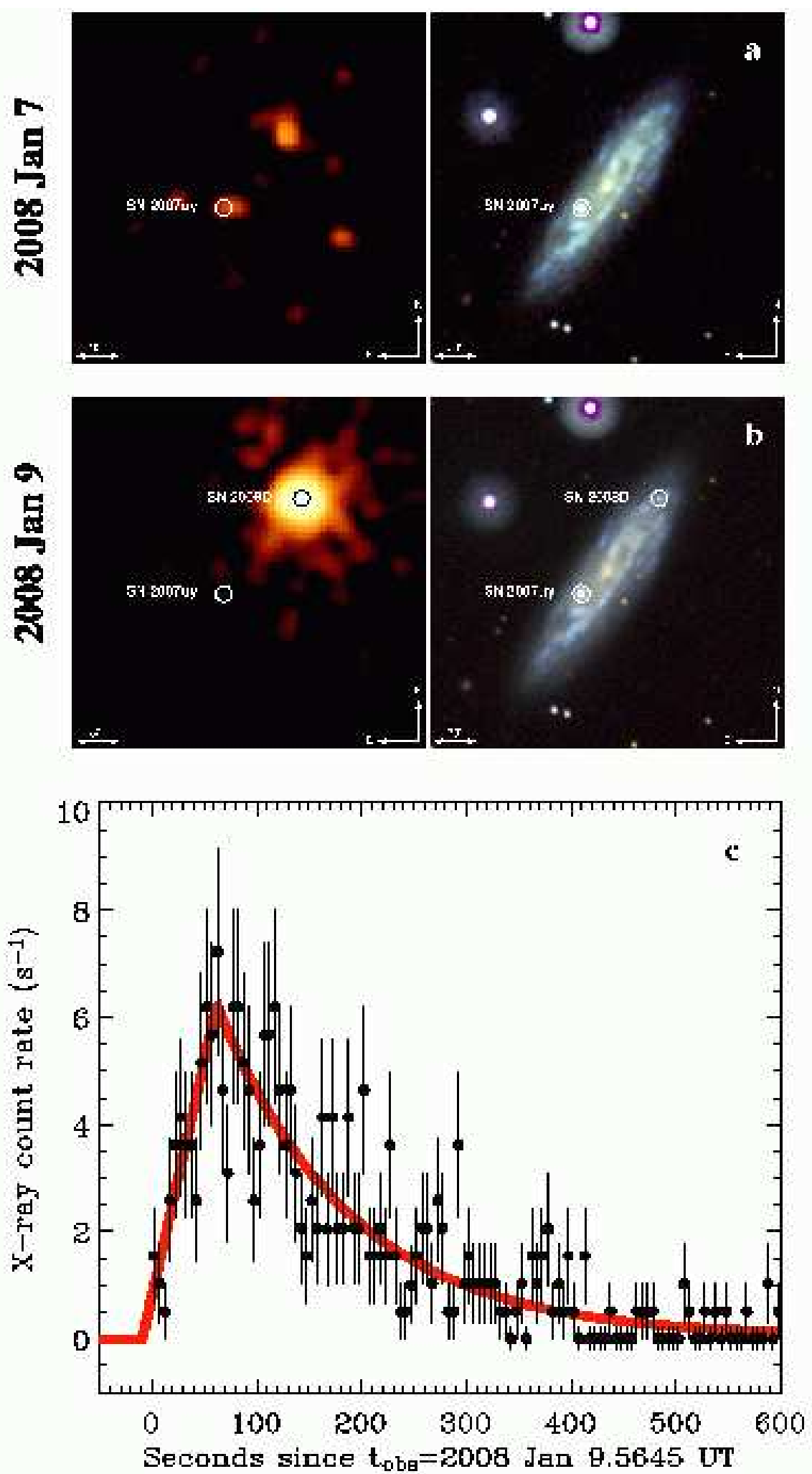

Figure 1. 


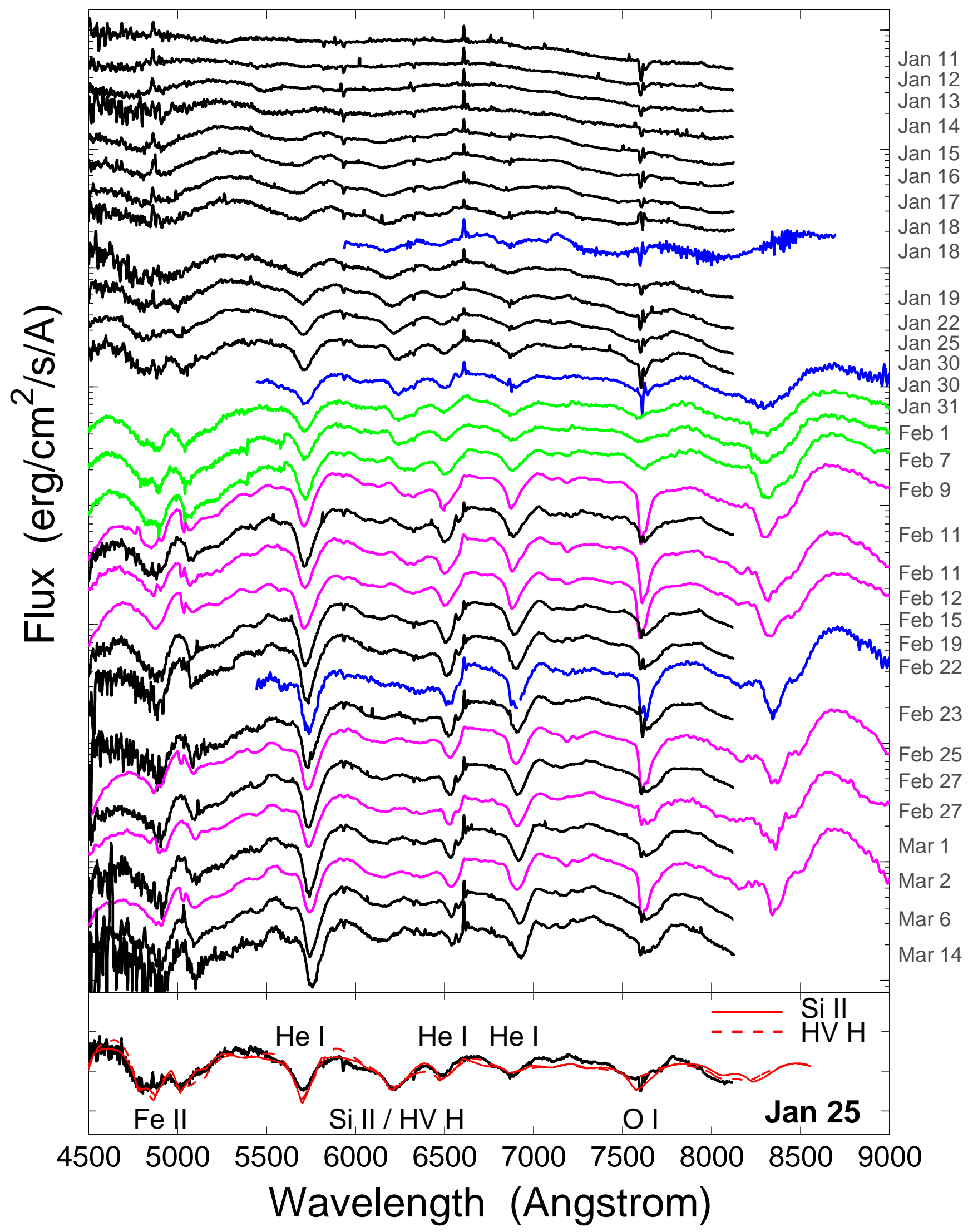

Figure 2. 


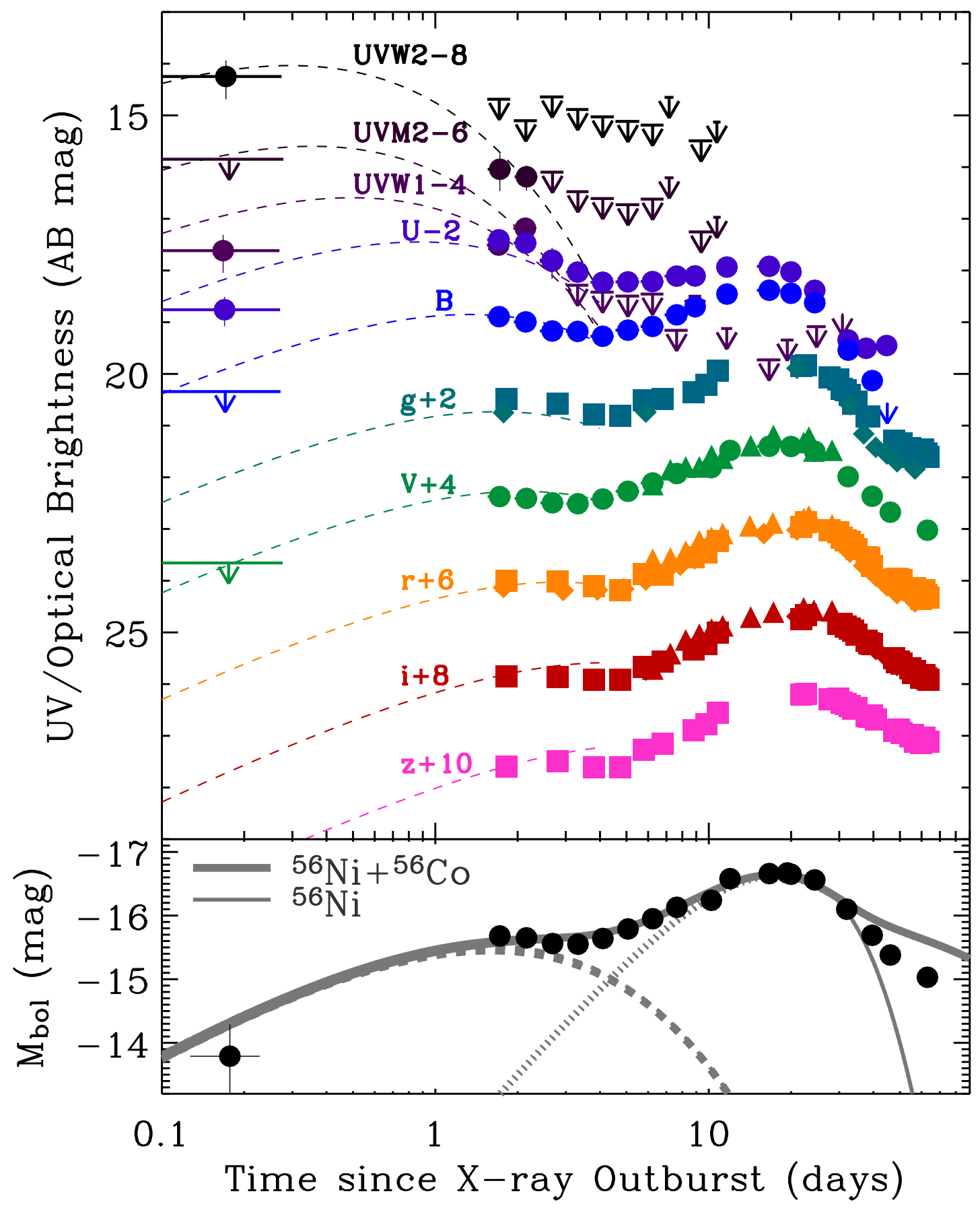

Figure 3. 
20 Soderberg et al.
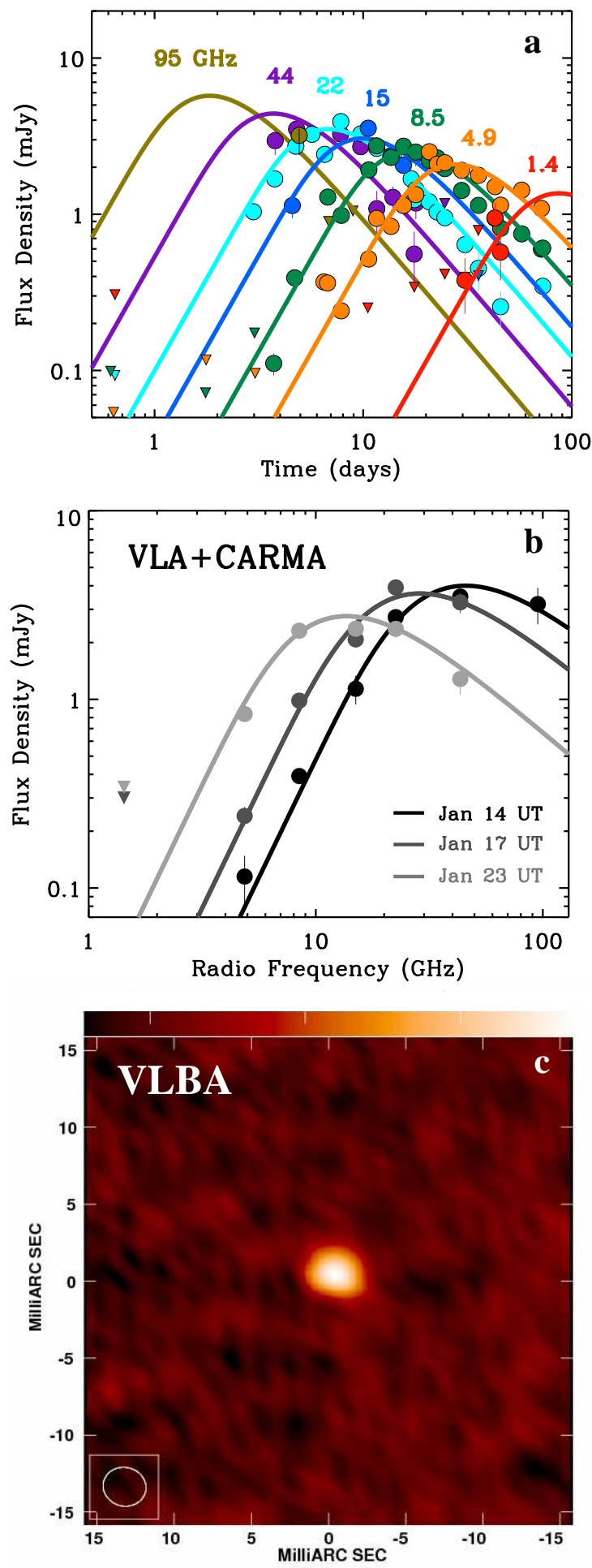

Figure 4. 


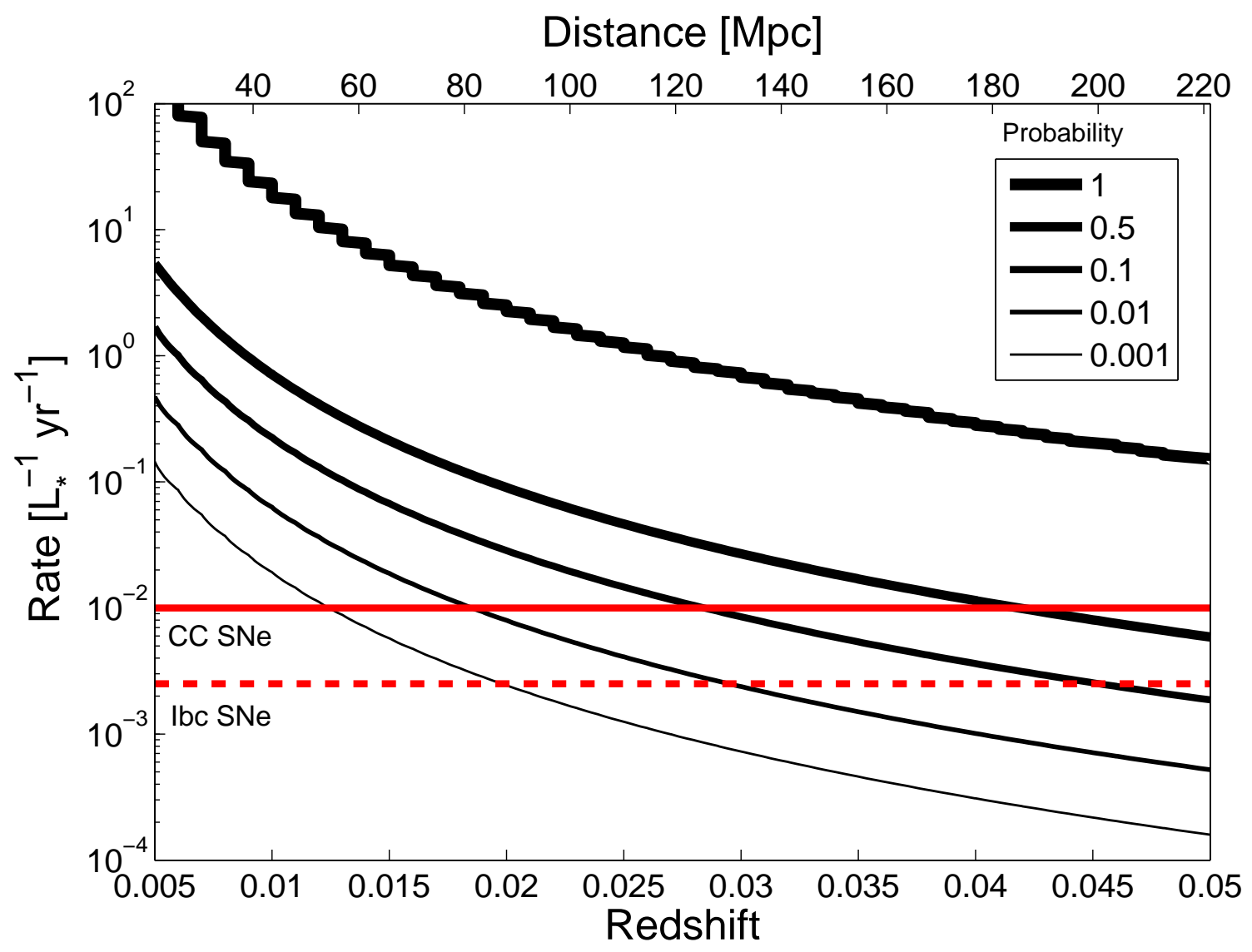

Figure 5. 